\title{
Acoustic analog of Hawking radiation in quantized circular superflows of Bose-Einstein condensates
}

\author{
Igor Yatsuta $\odot,{ }^{1}$ Boris Malomed $\odot,{ }^{2,3}$ and Alexander Yakimenko $\oplus^{1}$ \\ ${ }^{1}$ Department of Physics, Taras Shevchenko National University of Kyiv, 64/13, Volodymyrska Street, Kyiv 01601, Ukraine \\ ${ }^{2}$ Department of Physical Electronics, School of Electrical Engineering, Faculty of Engineering, and Center for Light-Matter Interaction, \\ Tel Aviv University, 69978 Tel Aviv, Israel \\ ${ }^{3}$ Instituto de Alta Investigación, Universidad de Tarapacá, Casilla 7D, Arica, Chile
}

(Received 23 June 2020; accepted 23 September 2020; published 12 October 2020)

\begin{abstract}
We propose emulation of Hawking radiation (HR) by means of acoustic excitations propagating on top of persistent current in an atomic Bose-Einstein condensate (BEC) loaded in an annular confining potential. The setting is initially created as a spatially uniform one, and then switches into a nonuniform configuration, while maintaining uniform BEC density. The eventual setting admits the realization of sonic black and white event horizons with different slopes of the local sound speed. A smooth slope near the white-hole horizon suppresses instabilities in the supersonic region. It is found that tongue-shaped patterns of the density-density correlation function, which represent the acoustic analog of HR, are strongly affected by the radius of the ring-shaped configuration and number of discrete acoustic modes admitted by it. There is a minimum radius that enables the emulation of HR. We also briefly discuss a possible similarity of properties of the matter-wave sonic black holes to the known puzzle of the stability of Planck-scale primordial black holes in quantum gravity.
\end{abstract}

DOI: 10.1103/PhysRevResearch.2.043065

\section{INTRODUCTION}

Black holes (BHs) are among the most fascinating objects in the Universe, the study of which may help us to illuminate an intimate relation between gravity and the quantum theory. Pioneering works [1-4] of Bekenstein and Hawking had allowed elucidating the remarkable quantum properties of BHs. It was discovered that a $\mathrm{BH}$ is not a stationary everlasting object, as it emits black-body radiation. The effective temperature of the Hawking radiation (HR) is extremely low for known astrophysical BHs, which makes it practically impossible to observe the emission effect, therefore attention has turned to settings that admit emulation of this phenomenology in other physical settings.

Acoustic BHs were first introduced by Unruh [5,6], who had demonstrated precise formal equivalence between the behavior of sound waves in fluid flow and that of a scalar field in curved space-time. Thus, it is possible to create an analog BH in superfluids where the transition from subsonic to supersonic flow plays the role of the event horizon. Following that work, many experimental realizations were proposed for demonstrating acoustic horizons. Behaviors similar to the ana$\log$ HR have been experimentally and theoretically explored in trapped ions [7], optical fibers [8-11], electromagnetic waveguides [12], water tanks [13,14], ultracold fermions [15], exciton-polariton condensates [16], and superfluid ${ }^{3} \mathrm{He}$ [17].

Published by the American Physical Society under the terms of the Creative Commons Attribution 4.0 International license. Further distribution of this work must maintain attribution to the author(s) and the published article's title, journal citation, and DOI.
Tremendous progress in physics of ultracold atomic gases [18] makes atomic Bose-Einstein condensates (BECs) very suitable testbeds for sonic BHs [19,20]. In these connections, recent experimental realization [21-24] of analogs of the HR in atomic $\mathrm{BEC}$ is an important milestone in the emulation of quantum properties of BHs.

Most previous works addressing sonic BHs in BECs were focused on cigar-shaped quasi-one-dimensional (1D) condensates [22], or ones subject to specific absorbing boundary conditions used for suppression of spurious instabilities $[19,20]$, which gives rise to a single $\mathrm{BH}$ horizon. On the other hand, toroidal BECs were used to emulate quantum features of $\mathrm{HR}$, as the angular momentum is quantized in the ring-shaped BEC due to periodic boundary conditions. In this work we address quasi-1D atomic BEC confined in a toroidal trap. This setting, which is available in experiments [25-29], was used for studies of diverse matter-wave patterns maintained by circular flows, i.e., effects induced by the angular momentum [30-44].

Previous investigations $[45,46]$ of the event horizon in BEC with toroidal geometry relied upon the use of the supersonic flow driven by a variable-density distribution in the condensate. A spectrum of the eigenstates on top of the nonuniform background was found in Ref. [46], demonstrating parametric amplification (i.e., dynamical instabilities) at certain frequencies, and it was compared to the two-mode approximation $[45,46]$. Significant theoretical progress in studies of acoustic analogs of HR was achieved with the help of the correlationfunction method, which was extensively used in the context of elongated 1D condensates [19,20,22,23]. However, not much attention was paid to the white-hole (WH) horizon, and the role of the spatial slope of the nonuniform local parameters of the condensates near this point, on the stability of the system. 


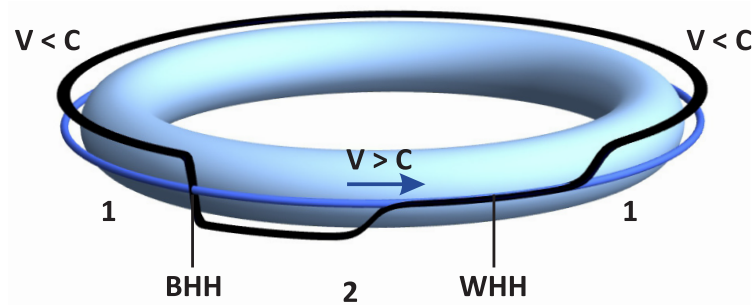

FIG. 1. A schematic of the ring-shaped Bose-Einstein condensate with asymmetric acoustic event horizons. Shown are the blue isosurface of the azimuthally uniform condensate density $n$, and the constant superflow velocity $v$ (the dark blue line). The azimuthally modulated interaction strength $g(x, t)$ is responsible for the variation of the local sound speed $c(x)$ (the black line), which identifies the subsonic $(1: v<c)$ and supersonic $(2: v>c)$ regions. The arrow shows the direction of the persistent current. Acoustic black-hole and white-hole horizons (BHH and $\mathrm{WHH}$, respectively) are located at points with $c(x)=v$.

Here we consider the setting with a uniform condensate density, while the event horizon is created by spatiotemporal modulation of the coupling constant, which is responsible for interatomic interactions in the mean-field approximation $[18,19]$. The coexistence of $\mathrm{BH}$ and $\mathrm{WH}$ event horizons in the toroidal geometry gives rise to amplified HR and formation of background ripples [47-51]. Extensive investigation of related instabilities by dint of the correlation-function method has been carried out in the 1D system with absorbing boundary conditions [52]. Here we focus on the HR per se, rather than the amplification effect induced by the instability. As is known $[6,23,53]$, the analog Hawking temperature for the effectively $1 \mathrm{D}$ flow is

$$
T_{H}=\left.\frac{\hbar}{2 \pi k_{B}}\left(\frac{\partial v}{\partial x}-\frac{\partial c}{\partial x}\right)\right|_{x=x_{h}},
$$

where $v(x)$ is the local velocity of the condensate, $c(x)$ is the local speed of sound, and the derivatives are evaluated at the position of the horizon, $x=x_{h}$. To minimize the impact of the WH horizon, we introduce a steep gradient of $c(x)$ near the $\mathrm{BH}$ horizon, and a smoothed gradient near the WH one, as shown in Fig. 1. This setting makes it possible to conveniently manipulate the slopes, $\partial v / \partial x$ and $\partial c / \partial x$ in Eq. (1), while keeping parameters of the acoustic $\mathrm{BH}$ horizon and density of the condensate unchanged.

The main objective of the present work is the impact of the quantization of the superflow, imposed by the periodic boundary conditions, on the analog HR in the toroidal BEC. The analysis reveals the following noteworthy effects. (i) A smooth WH horizon suppresses instabilities and makes the supersonic flow robust. (ii) Density-density correlations, representing the acoustic analog of HR, are strongly affected by the size of the ring's radius and the number of discrete acoustic modes available in the annular geometry initially. (iii) HR vanishes if the radius falls below a critical value.

The rest of the paper is organized as follows. The model is introduced in Sec. II. In Sec. III we discuss initial fluctuations and the corresponding dispersion relation. In Sec. IV we present results for density-density correlations and the acoustic analog of HR near the $\mathrm{BH}$ horizon. The paper is concluded in Sec. V, where we also discuss possible similarities of properties of the analog $\mathrm{BHs}$ to some fundamental problems, such as stability of primordial quantum BHs on the Planck's scale.

\section{THE MODEL}

We address the acoustic analog of HR in the framework of the 1D Gross-Pitaevskii equation (GPE), written in scaled units,

$$
i \frac{\partial \psi}{\partial t}=\left[-\frac{1}{2} \frac{\partial^{2}}{\partial x^{2}}+V(x, t)+g(x, t)|\psi|^{2}\right] \psi,
$$

where $\psi(x+L, t)=\psi(x, t)$ is the wave function of the ringshaped condensate of ring $R$ and length $L=2 \pi R$, while $V(x, t)$ and $g(x, t)$ are the effective potential and 1D coupling constant, respectively. An efficient method for detecting the analog HR is the analysis of density-density correlations [54-56]. We here use mean-field simulations of the GPE, together with a sampling of the quantum noise by means of a procedure similar to the well-known truncated Wigner approximation (TWA) [57-61]. In our analysis of the quantum fluid dynamics, we consider the evolution of stochastic trajectories satisfying the 1D GPE (2). Similar to the standard TWA, we add extra noise to the initial wave function, thus taking it as follows:

$$
\psi(x, 0)=e^{i k_{0} x}\left[\sqrt{n_{0}}+\frac{1}{\sqrt{L}} \sum_{k \neq 0}\left(\beta_{k} u_{0 k} e^{i k x}+\beta_{k}^{*} w_{0 k}^{*} e^{-i k x}\right)\right],
$$

where $k_{0}=M v / \hbar$ is determined by the flow velocity $v, \beta_{k}, \beta_{k}^{*}$ are noise amplitudes taking random values, the summation is performed over wave numbers $k, n_{0}$ is the spatially uniform density, and $u_{0 k}, w_{0 k}$ are defined as

$$
u_{0 k}, w_{0 k}=\left[\left(E_{k} / \epsilon_{k}\right)^{1 / 2} \pm\left(E_{k} / \epsilon_{k}\right)^{-1 / 2}\right] / 2,
$$

where $E_{k} \equiv k^{2} / 2, \epsilon_{k} \equiv \sqrt{E_{k}\left(E_{k}+2 g n\right)}$.

It is relevant to briefly discuss the derivation of GPE (2) and the meaning of its parameters, as well as the meaning of the noise in Eq. (3). As said above, we consider the system schematically shown in Fig. 1. The azimuthally modulated interaction strength $g(x, t)$, where $x=R \varphi$ and $\varphi$ is the azimuthal coordinate along the ring, is responsible for the variation of the local sound speed $c(x)$. The starting point of the derivation is the $3 \mathrm{D}$ mean-field GPE in physical units:

$$
i \hbar \frac{\partial \bar{\Psi}}{\partial \bar{t}}=\left[-\frac{\hbar^{2}}{2 M} \bar{\nabla}^{2}+\bar{V}_{e}(\overline{\mathbf{r}}, \bar{t})+\gamma(\overline{\mathbf{r}}, \bar{t})|\bar{\Psi}|^{2}\right] \bar{\Psi},
$$

where $M$ is the atomic mass, $\bar{V}_{e}=\bar{V}_{\text {trap }}+\bar{V}_{h}$ is the external potential that consists of two terms, viz., the transverse confinement and the potential that creates the horizon, $\gamma=$ $4 \pi \hbar^{2} a_{s} / M$ is the coupling constant, and $a_{s}$ is the $s$-wave scattering length, which is supposed to be a function of the coordinate and time. The 3D wave function $\bar{\Psi}(\overline{\mathbf{r}}, \bar{t})$ determines the number of atoms $N$ by the normalization condition $N=$ $\int|\bar{\Psi}|^{2} d^{3} \overline{\mathbf{r}}$. We fix $N=10^{5}$ of ${ }^{87} \mathrm{Rb}$ atoms in our calculations.

Thus, we consider a thin ring of radius $\bar{R}$ filled by dilute BEC, with the transverse degrees of freedom frozen by 
the tight confinement. Assuming the usual factorized ansatz [30,62-64], we set

$$
\bar{\Psi}(\overline{\mathbf{r}}, \bar{t})=\bar{\psi}(\bar{x}, \bar{t}) \bar{\phi}\left(\overline{\mathbf{r}}_{\perp}, \bar{t}\right),
$$

where $\bar{\phi}\left(\overline{\mathbf{r}}_{\perp}\right)$ is the function of transverse coordinates (see, e.g., [65]), and the 1D density is normalized to the total number of atoms:

$$
\int d^{2} \overline{\mathbf{r}}_{\perp}|\bar{\phi}|^{2}=1, \quad \bar{n} \equiv|\bar{\psi}|^{2}=\int d^{2} \overline{\mathbf{r}}_{\perp}|\bar{\Psi}|^{2} .
$$

Then, averaging Eq. (5) in the transverse plane leads to the effective 1D GPE (2) with the 1D coupling constant defined as

$$
\bar{g}=\frac{4 \pi \hbar^{2} a_{s}}{M} \int d^{2} \overline{\mathbf{r}}_{\perp}|\bar{\phi}|^{4} .
$$

The state of the condensate in the ring with the uniform density is provided by the plane-wave solution [19] of Eq. (2):

$$
\bar{\psi}=\sqrt{\bar{n}_{0}} e^{i\left(\bar{k}_{0} \bar{x}-\bar{\omega}_{0} \bar{t}\right)}, \quad \hbar \bar{\omega}_{0}=\frac{\left(\hbar \bar{k}_{0}\right)^{2}}{2 M}+\bar{V}(\bar{x}, \bar{t})+\bar{g}(\bar{x}, \bar{t}) \bar{n}_{0} .
$$

Potential $\bar{V}(\bar{x}, \bar{t})$ and nonlinearity coefficient $\bar{g}(\bar{x}, \bar{t})$ must be mutually matched so as to admit the existence of the state with uniform density $\bar{n}_{0}$.

Since the wave function must be periodic, $\bar{\psi}(\bar{x})=\bar{\psi}(\bar{x}+$ $\bar{L}$ ), possible values of $\bar{k}_{0}$ and $\bar{k}$ in Eq. (3) are restricted by the length of the ring: $\left(\bar{k}_{0}, \bar{k}\right)=2 \pi\left(m_{0}, m\right) / \bar{L}$, with integers $m_{0}$ and $m$ determined by the obvious quantization of the velocity circulation:

$$
\oint \overline{\mathbf{v}} d \overline{\mathbf{l}}=2 \pi m \hbar / M
$$

where $m$ is the winding number, alias topological charge. If we solve Eq. (2) on a grid of $N_{p}$ points with spacing $\bar{h}_{p}$, then $\bar{k}_{0}=2 \pi m /\left(N_{p} \bar{h}_{p}\right)$, where integer $m$ coincides with the winding number.

A desirable spatial distribution of the local sound speeds in regions 1 and 2 in Fig. 1 can be produced by making the coupling constant, $\bar{g}$ in Eq. (5), spatially and temporally inhomogeneous. This can be implemented by either tuning the scattering length $a_{s}$ by means of the Feshbach resonance $[18,19,66-68]$, or longitudinally varying the strength of the transverse confinement [69]. The sound speed in regions 1 and 2 is $\bar{c}_{1,2}=\sqrt{\bar{\mu}_{1,2} / M}$, where $\bar{\mu}_{1,2}=\bar{g}_{1,2} \bar{n}$ are the effective local chemical potentials. For plane wave (9) with uniform density $\bar{n}_{0}$ to be a solution at all values of time, we need to adjust the axial potential and coupling constant in the two regions as follows:

$$
\bar{V}_{1}(\bar{x}, \bar{t})+\bar{g}_{1}(\bar{x}, \bar{t}) \bar{n}_{0}=\bar{V}_{2}(\bar{x}, \bar{t})+\bar{g}_{2}(\bar{x}, \bar{t}) \bar{n}_{0},
$$

where $-\bar{L} / 2<\bar{x}<\bar{L} / 2$. Actually, a part of the definition of the model is that the combination written in Eq. (11) remains constant over the ring.

Finally, to cast the 1D GPE in the normalized form of Eq. (2), we apply the following rescaling in both regions, and use the notation without bars: $t=\bar{t} / \bar{\tau}_{1}, \omega=\bar{\omega} \bar{\tau}_{1}, x=\bar{x} / \bar{\xi}_{1}$, $k=\bar{k} \bar{\xi}_{1}, \psi=\sqrt{\bar{\xi}_{1}} \bar{\psi}, g_{1,2}=\bar{g}_{1,2} /\left(\bar{\xi}_{1} \bar{\mu}_{1}\right)$, where $\bar{\tau}_{1}=\hbar / \bar{\mu}_{1}$, and $\bar{\xi}_{1} \equiv \sqrt{\hbar^{2} /\left(M \bar{\mu}_{1}\right)}$ is the healing length in region 1 .

The boundary conditions for Eq. (2) being periodic, we used the split-step fast Fourier transform method [70] for numerical simulations. The transition from $g_{1}, V_{1}$ to $g_{2}, V_{2}$ in region $\sigma_{x}$ is smooth, provided by the potential taken, locally, as

$$
V(x, t)=V_{1}+\Delta V(t) f\left(\frac{t-t_{0}}{\sigma_{t}}\right) f\left(\frac{x-x_{\mathrm{BHH}}}{\sigma_{x}}\right),
$$

where $-L / 2<x<0, \Delta V(t)=\left(V_{2}-V_{1}\right) \theta\left(t-t_{0}\right)[\theta(t)$ is the step function, hence $g$ and $V=V_{1}$ are constant at $t \leqslant 0$ ], $\sigma_{t}$ is the time of the switch of $V(x, t)$ and $g(x, t)$ from initial to final values, and the switching function is

$$
f(x)=\frac{1}{2}[1+\tanh (x / 2)] .
$$

Simultaneously with the variation of the potential, the nonlinearity coefficient was varied so as to keep $V(x, t)+g(x, t) n_{0}$ constant, see Eq. (11).

In our simulations we used $x_{\mathrm{BHH}}=-50$ and $t_{0}=5$. We have checked that the results do not change essentially if potential (12) is used without the jump imposed by $\theta\left(t-t_{0}\right)$, provided that condition $\sigma_{t} \ll t_{0}$ holds. The initial condition taken as the uniform condensate with constant $g$ and $V=V_{1}$ makes it easier to add the long-wavelength noise to the entire system or some part of it, if necessary.

Using this setting with variable of $V(x, t)$ and $g(x, t)$ defined as per Eq. (12) and running hundreds of simulations, it is straightforward to see that the initial noise added to the uniform condensate excites all possible long-wavelength modes admitted by nonuniform $g(x)$ and $V(x)$. We have checked that, for $\sigma_{t} \in[0.1,5]$, variation of this parameter does not change the dynamics qualitatively, therefore we here present the results for $\sigma_{t}=0.5$.

Handling the $\mathrm{WHH}$, which always appears in the ring, is more challenging, therefore we performed simulations for two different switching functions, the first one was chosen for $0<$ $x<L / 2$ as

$$
V(x, t)=V_{1}+\Delta V(t) f\left(\frac{t-t_{0}}{\sigma_{t}}\right) f\left(\frac{x_{\mathrm{WHH}}-x}{\sigma_{\mathrm{WHH}}}\right)
$$

that provides mirror symmetry for the global potential, under conditions $\sigma_{\mathrm{WHH}}=\sigma_{x}$ and $x_{\mathrm{WHH}}=-x_{\mathrm{BHH}}$, cf., Eq. (12). One can see that this type of the transition function cannot allow an arbitrary value of the slope of $c(x)$ at the WHH point, as it requires an extremely large length of the ring for large $\sigma_{\mathrm{WHH}}$ and small $|\partial c / \partial x| \sim\left(\sigma_{\mathrm{WHH}}\right)^{-1}$. To deal with this case, we used, instead of the switching scenario defined by Eq. (12), a double-step one, designed as a set of two similar potentials, see Figs. 3 and 4(b) below. These two functions gradually carry over into each other, allowing one to manipulate a sufficiently smooth slope near WHH.

Parameters of the horizon in the scaled units are similar to those in the model used in Ref. [19] and close to the experimental parameters of Ref. [22] for the speeds: $c_{1}=1$ $(1 \mathrm{~mm} / \mathrm{s}), v=0.74, c_{2}=0.5, \sigma_{x}=0.5, \sigma_{t}=0.5$, with the corresponding dispersion relations for regions far from the horizon shown in Fig. 2. Numerical values of $g_{1,2}$ and $V_{1,2}$ can be found from relation $\bar{g}=M \bar{c}^{2} / \bar{n}$ and $\bar{V}_{1}=1.6 \bar{\mu}_{1}$. The initial ring has the length of $\bar{L}=233 \mu \mathrm{m}$ with $\bar{\tau}_{1}=0.73 \mathrm{~ms}$, $\bar{\xi}_{1}=0.73 \mu \mathrm{m}, \bar{c}_{1}=1 \mathrm{~mm} / \mathrm{s}$, which is tantamount to $L=$ $320, \tau_{1}=1, \xi_{1}=1, \mu_{1}=1$, and $c_{1}=1$ in the scaled units. 

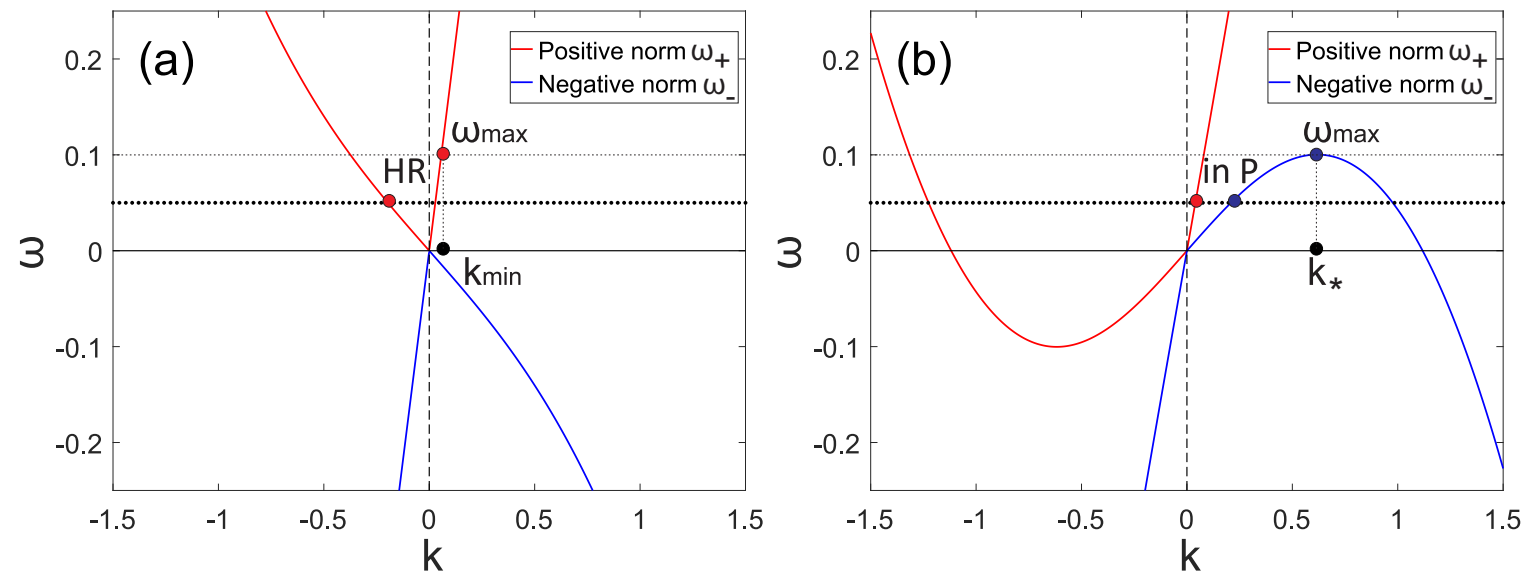

FIG. 2. The dispersion relation for regions far from the horizon. The dispersion curves (a) for $c=c_{1}$ (before BHH) and (b) for $c=c_{2}$ (behind BHH). (a) is relevant for the entire ring, until switching the horizon on at $t=5$. The HR (Hawking-radiation) mode moves against the flow. Positive- and negative-norm branches correspond to positive and negative signs of frequencies in the quiescent condensate. In the condensate with the flow in the supersonic region, the negative-norm branch acquires positive values for some values of $k$, which results in the emergence of the P (partner) mode. Modes P and "in" belong to different dispersion branches and move in the direction of the flow. They represent, respectively, the partner component in the Hawking pair, and a mode which exists even in the absence of the event horizon and moves towards the white-hole horizon. Constant $\omega_{\max }$ defines the frequency limit for the analog HR, representing the maximum frequency on the negative-norm branch in the supersonic region. Wave number $k_{\min }$ represents the restriction on the analog HR emission for modes with the positive direction of motion.

\section{QUANTUM FLUCTUATIONS AND THE DISPERSION RELATION}

In real BEC, the presence of quantum and thermal fluctuations, which are not taken into account by GPE, is inevitable. It is possible to include these effects by adding Gaussian noise to the initial wave function, according to TWA. The evolution of the fluctuations is governed by the Bogoliubov theory [18], and the expectation values of symmetrically ordered observables can be obtained by taking the stochastic average over the ensemble of evolved wave functions.

As said above, the initial state of the unperturbed system is uniform, and the potential step is switched on at time $t_{0}>$ 0 , therefore in our simulations we used the stochastic initial wave function (3), where the summation is performed up to some maximum wave number $k$. In the following section we discuss an appropriate choice of this limit value, and consider its impact on the analog HR.

It is straightforward to derive the respective dispersion relation for the acoustic waves, as small-amplitude collective perturbations running on top of the uniform-density potential:

$$
\omega_{ \pm}(k)=v k \pm \sqrt{\frac{k^{2}}{2}\left(\frac{k^{2}}{2}+2 g n_{0}\right)},
$$

where wave numbers $k$ should satisfy the same quantization condition as in wave function (9). To briefly explain the derivation of Eq. (15), we consider the wave function in a general form,

$$
\begin{aligned}
\psi(x, t) & =e^{-i \omega_{0} t}\left[\sqrt{n_{0}} e^{i k_{0} x}+\frac{1}{\sqrt{L}} \sum_{k \neq 0}\left[\beta_{k} u_{k}(x) e^{-i \omega_{k} t}\right.\right. \\
& \left.\left.+\beta_{k}^{*} w_{k}^{*}(x) e^{i \omega_{k} t}\right]\right]
\end{aligned}
$$

[cf., the initial condition given by Eq. (3)], where the perturbations are assumed to be small in comparison to $\sqrt{n_{0}}$, hence this expression can be rewritten as $\psi(x, t)=e^{-i \omega_{0} t}\left[\psi_{0}(x)+\right.$ $\left.\psi^{\prime}(x, t)\right]$, with $\left|\psi_{0}(x)\right| \gg\left|\psi^{\prime}(x, t)\right|$. After inserting this ansatz in GPE and linearizing with respect to $\psi^{\prime}(x, t)$, one derives a system of the Bogoliubov-de Gennes equations:

$$
\begin{aligned}
& \omega_{k} u_{k}(x)=\left(\hat{H}_{0}-\omega_{0}+2 g n\right) u_{k}(x)+g\left(\psi_{0}\right)^{2} w_{k}(x), \\
& \omega_{k} w_{k}(x)=\left(\hat{H}_{0}-\omega_{0}+2 g n\right) w_{k}(x)+g\left(\psi_{0}^{*}\right)^{2} u_{k}(x),
\end{aligned}
$$

where $\hat{H}_{0}=-1 / 2 \partial_{x x}^{2}+\hat{V}(x)$. Since $u_{k}(x)=u_{0 k} e^{i\left(k x+k_{0} x\right)}$, $w_{k}(x)=w_{0 k} e^{i\left(k x-k_{0} x\right)}$, and $\omega_{0}=k_{0}^{2} / 2+V+g n$, we obtain

$$
\begin{aligned}
& \left(\omega_{k}-k_{0} k-k^{2} / 2-g n\right) u_{0 k}-g n_{0} w_{0 k}=0, \\
& g n_{0} u_{0 k}+\left(\omega_{k}-k_{0} k+k^{2} / 2+g n\right) w_{0 k}=0 .
\end{aligned}
$$

It is easy to see that dispersion relation (15) follows from the consistency condition of system (18), setting $n=n_{0}$ in it. Coefficients $u_{0 k}, w_{0 k}(k \neq 0)$ are solutions of Eq. (18) such that the corresponding modes $u_{k}(x), w_{k}(x)$ satisfy the normalization condition $\int_{0}^{L}\left(\left|u_{k}(x)\right|^{2}-\left|w_{k}(x)\right|^{2}\right) d x=1$. Thus, expressions for amplitudes of the Bogoliubov modes take the form written in (4).

To keep the number of atoms constant, we adjusted density $n_{0}$ in each simulation. The expression that defines the number of excited atoms in the uniform condensate was produced in Ref. [59]. At zero temperature it can be written as

$$
N_{s}^{\prime}=\sum_{k \neq 0}\left(\left|u_{0 k}\right|^{2}+\left|w_{0 k}\right|^{2}\right)\left(\beta_{k}^{*} \beta_{k}-\frac{1}{2}\right)+\sum_{k \neq 0}\left|w_{0 k}\right|^{2} .
$$

Therefore, the number of atoms remaining in the ground states is $N_{c}=N-N_{s}^{\prime}$, and $n_{0}=\left(N_{c}+1 / 2\right) / L$. Such expressions follow from the relation between the average over ensemble and quantum average. According to Refs. [58,59], amplitudes 
(a)
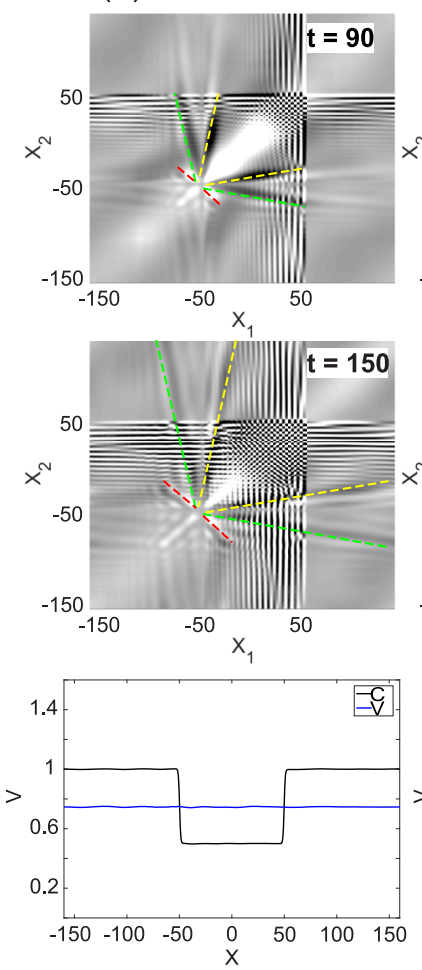

(b)
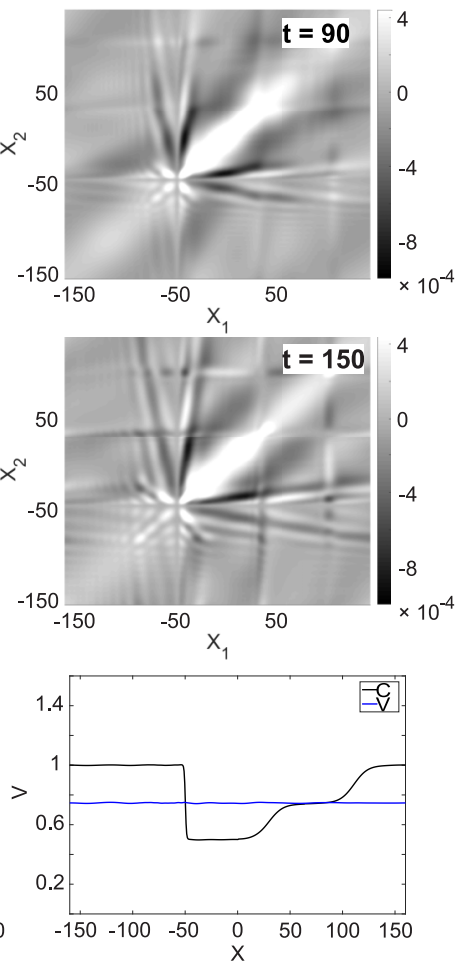

FIG. 3. Snapshots of the correlation function $n \xi_{1}\left[G\left(x_{1}, x_{2}\right)-1\right]$ for $t=90$ and $t=150$ for two different profiles of the local speed of sound with three initial modes and the ring's length $L=320$. The bottom row represents the distribution of the velocity (blue line) and local speed of sound (black curve) along the ring. (a) Symmetric BHH and WHH (black- and white-hole horizons) with sharp gradients of the local speed of sound near both horizons. (b) Speed of sound gradient is smoothed near the WHH. Note that the checkerboard pattern in the supersonic region, which is clearly seen in (a), vanishes in (b), when the local speed of sound gradually increases near the white horizon. Yellow, green, and red lines show expected positions of P-in, HR-in, and HR-P correlation tongues.

$\beta_{k}, \beta_{k}^{*}$ of the random perturbation are distributed as follows:

$$
W\left(\beta_{k}, \beta_{k}^{*}\right)=\frac{1}{2 \pi \sigma_{\epsilon}^{2}} \exp \left[-\frac{\left|\beta_{k}\right|^{2}}{2 \sigma_{\epsilon}^{2}}\right],
$$

where $\sigma_{\epsilon}=\left[4 \tanh \left(\epsilon_{k} / 2 k_{B} T_{0}\right)\right]^{-1 / 2}, T_{0}$ being the temperature of the condensate.

Clear evidence of the analog HR was obtained, both in the zero-temperature limit and for $T_{0}>0$, in Refs. [19,20]. Here we use the distribution for $T_{0}=0$ and focus on this fundamental case.

\section{ANALOG HR NEAR THE BHH}

To identify the generation of HR from the acoustic $\mathrm{BH}$, we have implemented the correlation-function method $[19,22,23,47,54,71-73]$. It was developed and used in Refs. [19,54] for the 1D condensate with a continuous transition between two uniform regions. The simple interpretation of this approach is that the HR and the corresponding partner particles are correlated with each other, although being located on opposite sides of the horizon. (a)
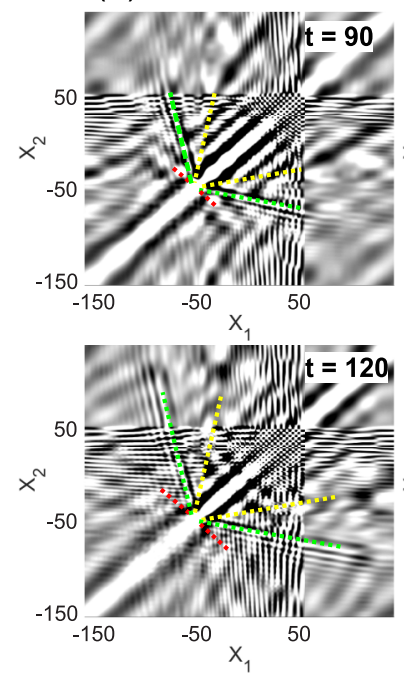

(b)
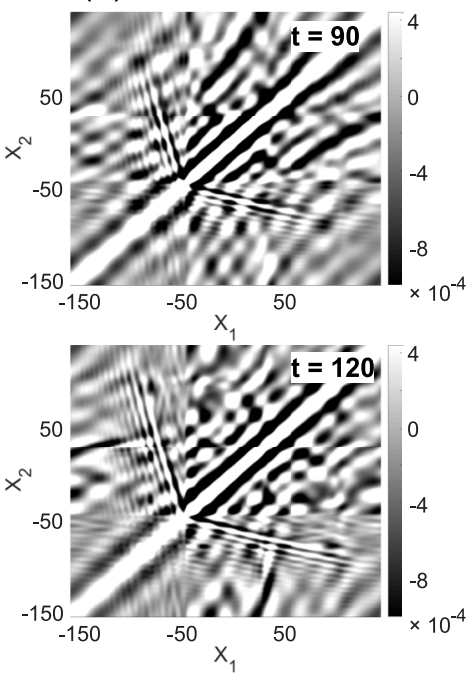

FIG. 4. The same as in Fig. 3 (without the bottom panels) for $L=320$, but inputs taken with ten initial modes. Note that the checkerboard pattern vanishes for the smoothed white horizon, as in Fig. 3.

We used the normalized density-density correlation function in the form of

$$
G\left(x_{1}, x_{2}\right)=\frac{\left\langle n\left(x_{1}\right) n\left(x_{2}\right)\right\rangle}{\left\langle n\left(x_{1}\right)\right\rangle\left\langle n\left(x_{2}\right)\right\rangle},
$$

where averaging is performed over an ensemble of $100 \mathrm{GPE}$ simulations. Further increase in the number of simulations does not tangibly affect the results. All simulations started with the input in the form of the uniform condensate with quantum noise added to it, as per Eq. (3). An essential role in the analysis is played by the number of modes which are used in TWA. The number was chosen to satisfy natural restrictions that allow one to observe signatures of the analog HR. Namely, TWA was proven to be correct for dilute Bose condensates if the number of modes obeys the constraint $N>N_{\text {modes }} / 2$ [58]. To provide the presence of $\mathrm{P}$ modes and thermality of the outgoing flux, frequencies should obey the condition

$$
\omega<\omega_{\max },
$$

see Fig. 2, as found in Ref. [74]. For a sufficiently low wave number, the latter condition also implies that all modes satisfy the linear dispersion relation, thus maintaining a clear analogy with $\mathrm{HR}$ near a real $\mathrm{BH}$.

Note that inequality (20) is written for the inhomogeneous setting in which the density is uniform, while $V$ and $g$ are not constant and both horizons are present. In this work we do not explicitly consider the eigenstate problem on top of the inhomogeneous background, but rather use eigenstates produced by the system of the Bogoliubov-de Gennes equations for the uniform condensates to generate initial random perturbations, as the initial background is uniform, see Eq. (12). By running multiple simulations of the ensuing evolution of the condensate for different realizations of the initial random perturbations, in the framework of the full GPE, we intend to produce all essential modes that can be excited after the 
switch to the inhomogeneous system at $t=t_{0}$. Accordingly, condition (20) was applied to the initial modes as an estimate for initial frequencies, which we expect to account for a qualitative change in the behavior of the correlation function. Therefore, below we refer to $\omega$ of the uniform setting and the respective dispersion relations displayed in Fig. 2.

The initial noise in Eq. (3) involves summation over negative and positive wave numbers $k$, but, in our investigation, only positive ones are relevant, as they correspond to modes moving towards BHH. Actually, including modes with the negative direction of motion did not produce any conspicuous change in the numerically computed correlations, therefore in what follows below the initial conditions include only lower positive- $k$ modes.

First, we address the symmetric potential with initial parameters given above $\left(L=320, x_{\mathrm{BHH}}=-50, x_{\mathrm{WHH}}=50\right.$, $\sigma_{x}=\sigma_{\mathrm{WHH}}=0.5$ ). Accordingly, in condition $\omega<\omega_{\max }$ we set $\omega_{\max } \approx 0.1$. In this case, the system admits three modes with the initial frequency below $\omega_{\max }$. The obtained results for the correlation function are displayed in Fig. 3. Colored lines in the figure correspond to the expected correlations between different particle pairs belonging to the dispersion relation in Fig. 2. The slope of the colored segment relative to the $x$ axis is determined by expressions for the sound speed of the Bogoliubov excitations:

$\tan \left(\theta_{y}\right)=\frac{v-c_{2}}{v+c_{2}}, \quad \tan \left(\theta_{g}\right)=\frac{v-c_{1}}{v+c_{2}}, \quad \tan \left(\theta_{r}\right)=\frac{v-c_{1}}{v-c_{2}}$,

where $\theta_{y}, \theta_{g}$, and $\theta_{r}$ refer to the yellow, green, and red lines, respectively, in Fig. 3. The length of each segment represents the expected length of the correlation tongues at the corresponding times, where "tongues" designate the line-shaped correlation regions that have one end located near BHH and the other end growing in time in a certain direction. It is seen that, in spite of the quantization of $k$, all the correlation tongues are visible for both potentials and they agree well with the predictions based on the dispersion relations. In Fig. 3 it is seen that the correlation pattern alters at later times [in Fig. 3(a) at $t \simeq 150$ ] because a checkerboard pattern appears as a result of the existence of a cavity between the horizons $[20,47]$. To resolve this problem, we decreased the value of $|\partial c / \partial x| \sim\left(\sigma_{\mathrm{WHH}}\right)^{-1}$, which is responsible for the mixing of modes at the horizon. Applying the double-step potential, we could make $|\partial c / \partial x|$ small enough, and thus avoid the destructive impact of such effects, by reducing the checkerboard correlations to just two crosses created by the scattering on each step. While we observe the correlations in both patterns for the current value of the ring's radius, the use of such decaying $|\partial c / \partial x|$ is necessary for smaller radii that are considered below.

The next step is to apply the initial noise, which contains modes that are located at $\omega>\omega_{\max }$, in terms of the dispersion relation. The corresponding correlations, produced by ten lowest modes, are shown in Fig. 4. As above, we see the same correlations for steep and smoothed slopes at WHH, with a qualitative difference in the checkerboard correlation patterns. Moreover, only HR-in correlation tongues (i.e., ones between excitation modes of the HR and in types) are present, and there is no evidence of P-in or HR-P tongues. We also note that obtained correlation tongues are expanding a bit faster than (a)
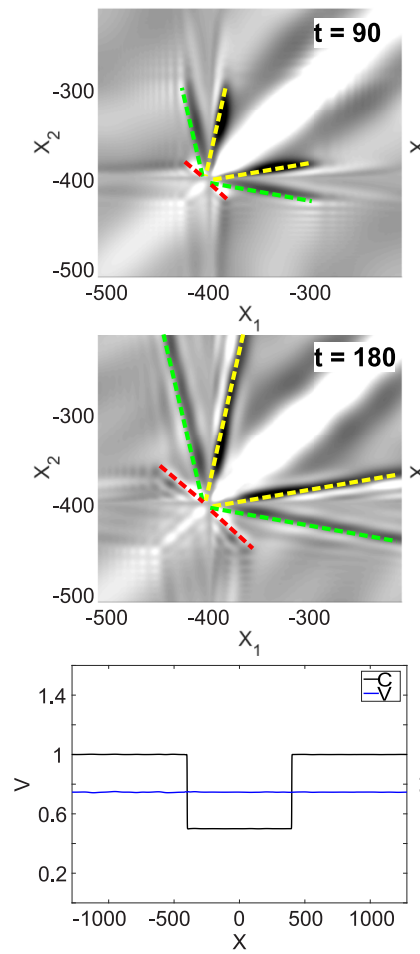

(b)
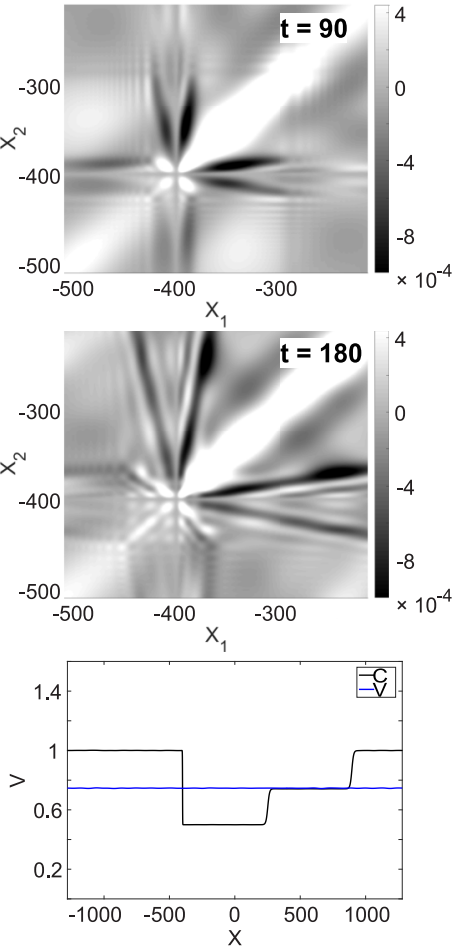

FIG. 5. Correlation function $n \xi_{1}\left[G\left(x_{1}, x_{2}\right)-1\right]$ for steep (a) and smoothed (b) slopes for 23 initial modes and $L=2560$. The bottom row shows velocity (blue line) and local speed of sound (black line) profiles near WHH. The spatial scale in the $\left(x_{1}, x_{2}\right)$ plane is the same as in Fig. 3.

expected, which may be a consequence of being beyond the linear-dispersion regime.

We have performed similar simulations for the eight times enlarged region and distance between the horizons $(L=2560$ or $\approx 1.87 \mathrm{~mm}$, in physical units), keeping the same parameters of both horizons as above. The larger area makes it possible to admits more initial modes that lie below $\omega_{\max }$. We have thus performed the simulations for 23 lowest modes belonging to the linear-dispersion regime. In Fig. 5 one can see the entire range of the expected correlations, and the expected positions of the tongues very well coinciding with the highlighted lines in the correlation pattern. These correlations feature relatively high intensity, which is about $4 \times 10^{-4}$ for the HR-P tongues. This value is still $\simeq 10$ times smaller than that reported in Ref. [19], which is explained by our choice of the diluteness parameter, $\xi_{1} n=1 / g_{1}=312.5 \gg 1$, that defines the intensity of the correlation signal. No drastic difference is seen between the correlations near BHH for both potential slopes at WHH. This fact is explained by a finite speed of excitations and relatively large distance between the two horizons.

The results obtained for the initial noise containing modes with $\omega<\omega_{\max }$ in a large ring $(L=2560)$ share main properties with the previous simulations: there is no difference between correlation patterns near BHH for two different slopes at WHH (at least in the course of the simulation time), and the correlation pattern, as one may expect, features solely HR-in correlation tongues. The same happens with modes that have energy above $\omega_{\max }$. 
The inference is that the possibility to observe the analog HR correlations (for fixed parameters of the flow and horizon) depends on the number of modes that have their frequencies below and above $\omega_{\max }$, as predicted by dispersion relation (15) for the uniform density. Furthermore, the effect is strongly affected by the size of the ring. The former aspect reflects the fact that, to produce the HR effect, one needs to have particles belonging to the frequency from the negative-norm branch of the dispersion relation in the laboratory reference frame. This criterion was also discovered analytically for the elongated 1D condensate with the single horizon [74], and it is true for our system as well, even if we apply it to the initial noise. The latter aspect implies that, by enlarging the length of the ring, one increases the number of allowed modes, which, in the limit of the ring with an infinite radius leads to the continuous frequency spectrum and an infinite number of modes at $\omega<\omega_{\max }$ past $t=t_{0}$. On the other hand, when the length of the ring decreases, the discreteness of the system's spectrum makes a great difference in the predicted observations: while some modes stay within the linear-dispersion region in a large-radius torus, the same set of the modes can partially or even completely exceed the frequency limit for a small ring. This change is accompanied by the transition from three pairs of the correlation tongues to the single surviving one, between the HR and "in" modes, or even the absence of any correlations, if a sufficiently large number of modes are located at $\omega>\omega_{\max }$. Lastly, it is expected that, in an elliptically deformed torus, the dependence of the HR effect on $L$ remains the same, in the first approximation.

Another important issue concerning our system with two horizons is its stability, which is sensitive to boundary conditions and the presence of the different horizons [75]. For the initial noise that does not strongly violate the frequency condition, we have found the steep horizon to be subject to eventual instability, with the respective lifetime $t_{\text {decay }} \simeq 600(\approx 0.438 \mathrm{~s}$, in physical units) for $L=320$. At larger times, simulations demonstrate that the development of the instability near WHH produces dark-soliton-like structures, and, finally, it leads to decay of both horizons. While similar behavior was reported in Ref. [45], in the present system instabilities grow near WHH, rather than BHH. On the other hand, the system with a smoothed horizon shows no instability (at least, in the course of long-time simulations for $t \leqslant 1 \mathrm{~s}$ ). The configurations in larger rings, with a bigger distance between the horizons, tend to be more stable than in smaller ones.

Due to the above-mentioned fact that the presence of the HR-P correlations tongue is determined by the number of initial modes below and above the critical value of $\omega_{\max }$, the value of the lowest wave number is restricted by the length of the ring. Therefore, there exists a critical size making it impossible to observe the HR correlations, as no Bogoliubov mode may satisfy the constraint (roughly speaking, this conclusion resembles the known property of the modulational instability, which is suppressed by periodic boundary conditions if the ring's length falls below the respective critical value [76]).

It is worth noting that restrictions on the size of the ring-shaped condensate have already been discussed in work [45]. That constraint originates from the phononic regime for the perturbations and sufficient conditions for the existence of the sonic horizon, giving critical value $R_{\mathrm{cr}}^{\prime}=\sqrt{2 \pi / N g}$ or $R_{1 \mathrm{cr}}^{\prime}\left(g_{1}\right)=0.14, R_{2 \mathrm{cr}}^{\prime}\left(g_{2}\right)=0.28$, which are significantly smaller than the critical radii obtained above. Moreover, $R_{\mathrm{cr}}^{\prime}$ depends on the particle number, which was kept constant in Ref. [45]. However, we change $N$ proportionally to $L$ in order to keep $n_{0}$ and $c_{1,2}=\sqrt{g_{1,2} n_{0}}$ constant. Consequently, our estimate for $R_{\mathrm{cr}}$ depends solely on parameters of the horizon, $v$ and $c_{1,2}$. The restriction on the radius is determined by the presence or absence of correlations as a signature of the analog-HR effect. The critical size $R_{\text {cr }}$ for our system can be evaluated from the condition that the frequency, which corresponds to the lowest wave number, pursuant to Eq. (15), is equal to $\omega_{\max }=\max \left[\omega_{-}\left(k ; g_{2}\right)\right]$, see Fig. 2 . The respective relation, which determines the critical radius $R_{\mathrm{cr}}=1 / k_{\mathrm{cr}}$, becomes

$$
\omega_{\max }=\frac{v}{R_{\mathrm{cr}}}+\sqrt{\frac{1}{2 R_{\mathrm{cr}}^{2}}\left(\frac{1}{2 R_{\mathrm{cr}}^{2}}+2 g_{1} n_{0}\right) .}
$$

For $1 / R_{\mathrm{cr}} \ll 1$ it simplifies to

$$
R_{\mathrm{cr}}=\frac{v+c_{1}}{\omega_{\max }}=\frac{2 v\left(v+c_{1}\right)}{2 k_{*}\left(v^{2}-c_{2}^{2}\right)-k_{*}^{3}},
$$

where

$$
k_{*} \equiv c_{2} \sqrt{-\left(2-\frac{v^{2}}{2 c_{2}^{2}}\right)+\frac{v}{c_{2}} \sqrt{2+\frac{v^{2}}{4 c_{2}^{2}}}} .
$$

It is relevant to compare the observed correlations for different critical radii evaluated from Eq. (22). With our initial parameters of $\mathrm{BHH}$, which allowed the observation of the HR-P correlations, the critical length of the ring is $L_{\mathrm{cr}} \approx 110$, which corresponds to critical radius $R_{\mathrm{cr}} \approx 17$ (or $\bar{R}_{\text {cr }} \approx 13 \mu \mathrm{m}$, in physical units). It is three times smaller than the radius of the ring in the simulations, $R \approx 51$. However, it remains a challenging objective to produce evidence of the disappearance of the HR-P correlations, as the noise with the strength used in the above simulations suppresses all correlations at $\bar{R} \simeq 13 \mu \mathrm{m}$. Therefore, we have changed parameters of the horizon to $v=0.61$ and $L_{\mathrm{cr}}=355$, so as to make the critical size of the ring slightly larger than $L=320$ (which is good to observe correlations), and conducted the simulations for a smoothed slope at WHH. In Fig. 6 we observe the absence of HR-P correlations for the three lowest modes. On the other hand, simulations produced visible P-in correlations (and negligible HR-P ones) for the ten lowest modes. Thus, we have performed numerical simulations of the nonuniform (at $t>t_{0}$ ) condensate and compared the results for the rings with the radius taken above and below the critical value $R_{\mathrm{cr}}$.

We stress that our consideration of the HR effect is based mostly on the direct numerical analysis of the density-density correlations. Surprisingly, predictions based on the oversimplified estimates of $R_{\mathrm{cr}}$, obtained for a uniform condensate, appears to be in good agreement with direct numerical simulations.

\section{CONCLUSIONS}

We have investigated the possibility to generate acoustic HR (Hawking radiation) in the superfluid ring-shaped BEC. For this purpose we have introduced the double-step potential 
(a)
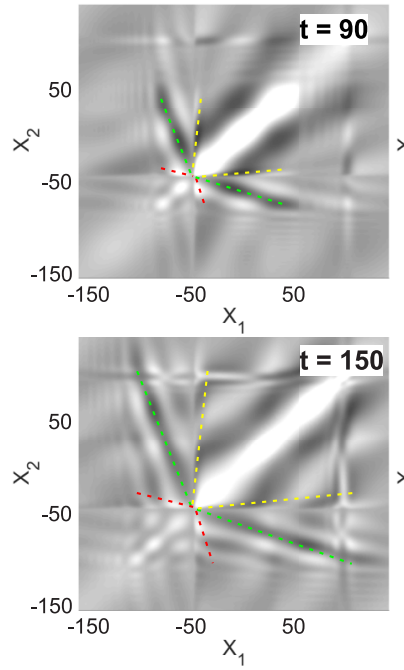

(b)
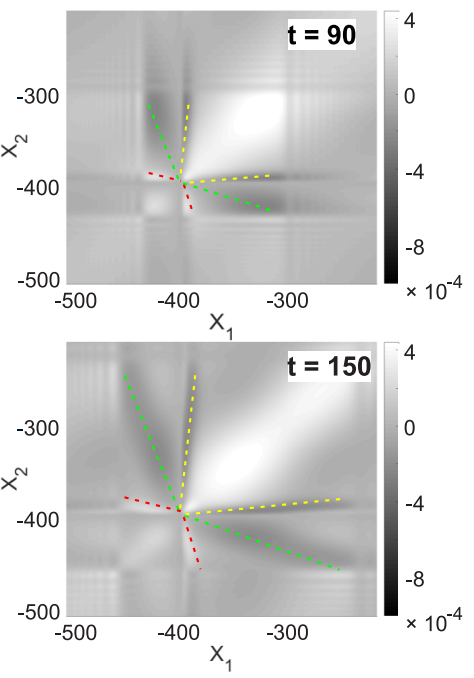

FIG. 6. Vanishing of HR-P correlations for the ring's length $L<$ $L_{\mathrm{cr}}$ for a ring with $v=0.61(m=31)$ and values of $|\partial c / \partial x|$ much smaller near WHH, in comparison to BHH. Column (a) pertains to the ring with three lowest modes and $L=320$, where even the lowest $k$ mode exceeds the frequency limit, and (b) provides snapshots of weak correlations for a larger ring $(L=2560)$ and ten lowest initial modes. The scale of the correlations is the same in all snapshots.

that minimizes the emulated Hawking temperature near the WHH (white-hole horizon), where instabilities may occur. The desirable region with the supersonic flow and uniform density distribution of the condensate may be designed using the spatiotemporally modulated interaction constant, adjusted to the selected potential. These features make the system considered here sufficiently stable and convenient for the analysis of the acoustic analog of HR in the rings.

We have addressed basic properties of the analog HR in the ring-shaped matter-wave configurations with different radii. The HR is quantified by the location and shape of tongueshaped correlation patterns, which, in turn, are well predicted by the dispersion relation for the quasiuniform condensate in the limit of low wave numbers. To this end, small random perturbations are added to the uniform system, in the framework of TWA (truncated Wigner approximation), and then multiple simulations of the full GPE are run for different realizations of the initial random perturbations, while the setting switches from spatially uniform to the nonuniform one. The so produced results for the correlation patterns are stable against variations of the spatiotemporal modulation of the potential and coupling constant.
An important circumstance is that the patterns are sensitive to the number of initial modes admitted by the ring, depending on its radius, below and above the frequency limit $\omega_{\max }$. Varying these parameters, we have observed different tongueshaped correlation patterns: from three pairs of tongues to a single one for a sufficiently large number of modes. The discreteness of the frequency and momentum spectra in the ring produces a dramatic effect on the properties of HR. Below the critical radius of the ring, even the lowest mode has its frequency above $\omega_{\max }$, so that HR-P correlations disappear. Thus, no acoustic HR takes place in the ring-shaped superflows with the radius falling below the critical value. Remarkably, a rough estimate for the critical radii, given by Eq. (22), which is obtained from the dispersion relation for the homogeneous condensate, agrees with results of direct numerical simulations of the perturbed dynamics of the inhomogeneous condensate.

It is relevant to compare the minimum radius of the ring, necessary for the generation of HR, with the situation for real (astrophysical) BHs (black holes). While they lose their mass through HR extremely slowly, the Hawking temperature dramatically increases for small BHs with mass $M: T_{H}=$ $\hbar c^{3} /\left(8 \pi G k_{B} M\right)=6.169 \times 10^{-8}\left(M_{\odot} / M\right) \mathrm{K}$. In this connection, it is relevant to mention that quantum effects are believed to be crucially important for BHs with the Planck-scale mass $M \sim m_{P}=\sqrt{\hbar c / G}$. A well-known puzzle in the quantum theory of gravity is the final fate of such BHs. There are good grounds to assume [77] that HR is suppressed for sufficiently small BHs when their size $r_{g}=2 G M / c^{2}$ becomes comparable to the Compton wavelength $\lambda_{C}=h /\left(m_{P} c\right)$, associated with the BH of the Planck's mass. On the other hand, we have found that there is also a critical size of the system, related to quantum effects in the toroidal geometry. The critical size depends on the spatial structure of the superflow near the $\mathrm{BH}$ horizon, which determines the strength of the analog "surface gravity." Certainly, the properties caused by the quantization, i.e., the discreteness of the frequency and momentum spectra, become essential when the size of the ring shrinks at a fixed value of the slope of the speed of sound. When the system's radius falls below the critical value $R_{\mathrm{cr}}$, the analog HR disappears. Accordingly, in terms of real BHs, small nonradiating primordial black holes, which are conjectured to be created in great numbers in the early Universe, might survive and become an ingredient of dark matter [78-81].

\section{ACKNOWLEDGMENT}

The work of B.A.M. is supported, in part, by the Israel Science Foundation through Grant No. 1286/17.
[1] J. D. Bekenstein, Black holes and entropy, Phys. Rev. D 7, 2333 (1973).

[2] S. W. Hawking, Black hole explosions? Nature (London) 248, 30 (1974).

[3] S. W. Hawking, Particle creation by black holes, Commun. Math. Phys. 43, 199 (1975).
[4] S. W. Hawking, Breakdown of predictability in gravitational collapse, Phys. Rev. D 14, 2460 (1976).

[5] W. G. Unruh, Notes on black-hole evaporation, Phys. Rev. D 14, 870 (1976).

[6] W. G. Unruh, Experimental Black-Hole Evaporation? Phys. Rev. Lett. 46, 1351 (1981). 
[7] B. Horstmann, B. Reznik, S. Fagnocchi, and J. I. Cirac, Hawking Radiation from an Acoustic Black Hole on an Ion Ring, Phys. Rev. Lett. 104, 250403 (2010).

[8] T. G. Philbin, C. Kuklewicz, S. Robertson, S. Hill, F. König, and U. Leonhardt, Fiber-optical analog of the event horizon, Science 319, 1367 (2008).

[9] F. Belgiorno, S. L. Cacciatori, M. Clerici, V. Gorini, G. Ortenzi, L. Rizzi, E. Rubino, V. G. Sala, and D. Faccio, Hawking Radiation from Ultrashort Laser Pulse Filaments, Phys. Rev. Lett. 105, 203901 (2010).

[10] W. G. Unruh and R. Schützhold, Hawking radiation from "phase horizons" in laser filaments? Phys. Rev. D 86, 064006 (2012).

[11] S. Liberati, A. Prain, and M. Visser, Quantum vacuum radiation in optical glass, Phys. Rev. D 85, 084014 (2012).

[12] R. Schützhold and W. G. Unruh, Hawking Radiation in an Electromagnetic Waveguide? Phys. Rev. Lett. 95, 031301 (2005).

[13] S. Weinfurtner, E. W. Tedford, M. C. J. Penrice, W. G. Unruh, and G. A. Lawrence, Measurement of Stimulated Hawking Emission in an Analog System, Phys. Rev. Lett. 106, 021302 (2011).

[14] G. Rousseaux, C. Mathis, P. Maïssa, T. G. Philbin, and U. Leonhardt, Observation of negative-frequency waves in a water tank: A classical analogue to the Hawking effect? New J. Phys. 10, 053015 (2008).

[15] S. Giovanazzi, Hawking Radiation in Sonic Black Holes, Phys. Rev. Lett. 94, 061302 (2005).

[16] D. D. Solnyshkov, H. Flayac, and G. Malpuech, Black holes and wormholes in spinor polariton condensates, Phys. Rev. B 84, 233405 (2011).

[17] T. A. Jacobson and G. E. Volovik, Event horizons and ergoregions in ${ }^{3}$ He, Phys. Rev. D 58, 064021 (1998); U. R. Fischer and G. E. Volovik, Thermal quasi-equilibrium states across landau horizons in the effective gravity of superfluids, Int. J. Modern Phys. D 10, 57 (2001).

[18] L. Pitaevskii and S. Stringari, Bose-Einstein Condensation and Superfluidity (Oxford University Press, New York, 2016).

[19] I. Carusotto, S. Fagnocchi, A. Recati, R. Balbinot, and A. Fabbri, Numerical observation of Hawking radiation from acoustic black holes in atomic Bose Einstein condensates, New J. Phys. 10, 103001 (2008).

[20] C. Mayoral, A. Recati, A. Fabbri, R. Parentani, R. Balbinot, and I. Carusotto, Acoustic white holes in flowing atomic BoseEinstein condensates, New J. Phys. 13, 025007 (2011).

[21] O. Lahav, A. Itah, A. Blumkin, C. Gordon, S. Rinott, A. Zayats, and J. Steinhauer, Realization of a Sonic Black Hole Analog in a Bose-Einstein Condensate, Phys. Rev. Lett. 105, 240401 (2010).

[22] J. Steinhauer, Observation of quantum Hawking radiation and its entanglement in an analogue black hole, Nat. Phys. 12, 959 (2016).

[23] J. R. Muñoz de Nova, K. Golubkov, V. I. Kolobov, and J. Steinhauer, Observation of thermal Hawking radiation and its temperature in an analogue black hole, Nature (London) 569, 688 (2019).

[24] A. Fabbri and R. Balbinot, Ramp-up of Hawking radiation in BEC analogue black holes, arXiv:2005.04443.

[25] S. Gupta, K. W. Murch, K. L. Moore, T. P. Purdy, and D. M. Stamper-Kurn, Bose-Einstein Condensation in a Circular Waveguide, Phys. Rev. Lett. 95, 143201 (2005).
[26] A. S. Arnold, C. S. Garvie, and E. Riis, Large magnetic storage ring for Bose-Einstein condensates, Phys. Rev. A 73, 041606(R) (2006).

[27] B. E. Sherlock, M. Gildemeister, E. Owen, E. Nugent, and C. J. Foot, Time-averaged adiabatic ring potential for ultracold atoms, Phys. Rev. A 83, 043408 (2011).

[28] A. Ramanathan, K. C. Wright, S. R. Muniz, M. Zelan, W. T. Hill III, C. J. Lobb, K. Helmerson, W. D. Phillips, and G. K. Campbell, Superflow in a Toroidal Bose-Einstein Condensate: An Atom Circuit with a Tunable Weak Link, Phys. Rev. Lett. 106, 130401 (2011).

[29] R. Bürkle, A. Gaidoukov, and J. R. Anglin, Quasi-steady radiation of sound from turbulent sonic ergoregions, New J. Phys. 20, 083020 (2018)

[30] A. Parola, L. Salasnich, R. Rota, and L. Reatto, Quantum phases of attractive matter waves in a toroidal trap, Phys. Rev. A 72, 063612 (2005).

[31] S. Schwartz, M. Cozzini, C. Menotti, I. Carusotto, P. Bouyer, and S. Stringari, One-dimensional description of a Bose Einstein condensate in a rotating closed-loop waveguide, New J. Phys. 8, 162 (2006).

[32] I. Lesanovsky and W. von Klitzing, Spontaneous Emergence of Angular Momentum Josephson Oscillations in Coupled Annular Bose-Einstein Condensates, Phys. Rev. Lett. 98, 050401 (2007).

[33] A. V. Carpentier and H. Michinel, A ring accelerator for matterwave solitons, Europhys. Lett. 78, 10002 (2007).

[34] J. Smyrnakis, S. Bargi, G. M. Kavoulakis, M. Magiropoulos, K. Kärkkäinen, and S. M. Reimann, Mixtures of Bose Gases Confined in a Ring Potential, Phys. Rev. Lett. 103, 100404 (2009).

[35] J. Brand, T. J. Haigh, and U. Zülicke, Rotational fluxons of Bose-Einstein condensates in coplanar double-ring traps, Phys. Rev. A 80, 011602(R) (2009).

[36] J. Smyrnakis, M. Magiropoulos, G. M. Kavoulakis, and A. D. Jackson, Solitary waves in mixtures of Bose gases confined in annular traps, Phys. Rev. A 81, 063601 (2010).

[37] S. Baharian and G. Baym, Bose-Einstein condensates in toroidal traps: Instabilities, swallow-tail loops, and selftrapping, Phys. Rev. A 87, 013619 (2013).

[38] A. I. Yakimenko, Yu. M. Bidasyuk, O. O. Prikhodko, S. I. Vilchinskii, E. A. Ostrovskaya, and Yu. S. Kivshar, Optical tweezers for vortex rings in Bose-Einstein condensates, Phys. Rev. A 88, 043637 (2013).

[39] M. Abad, A. Sartori, S. Finazzi, and A. Recati, Persistent currents in two-component condensates in a toroidal trap, Phys. Rev. A 89, 053602 (2014).

[40] M. Cominotti, D. Rossini, M. Rizzi, F. Hekking, and A. Minguzzi, Optimal Persistent Currents for Interacting Bosons on a Ring with a Gauge Field, Phys. Rev. Lett. 113, 025301 (2014).

[41] D. Gallucci and N. P. Proukakis, Engineering dark solitary waves in ring-trap Bose-Einstein condensates, New J. Phys. 18, 025004 (2016).

[42] Z. Chen, Y. Li, N. P. Proukakis, and B. A. Malomed, Immiscible and miscible states in binary condensates in the ring geometry, New J. Phys. 21, 073058 (2019).

[43] T. Bland, Q. Marolleau, P. Comaron, B. A. Malomed, and N. P. Proukakis, Persistent current formation in double-ring geometries, J. Phys. B: At., Mol. Opt. Phys. 53, 115301 (2020). 
[44] P. B. Walczak and J. R. Anglin, Exact Bogoliubov-de Gennes solutions for gray-soliton backgrounds, Phys. Rev. A 84, 013611 (2011).

[45] L. J. Garay, J. R. Anglin, J. I. Cirac, and P. Zoller, Sonic black holes in dilute Bose-Einstein condensates, Phys. Rev. A 63, 023611 (2001).

[46] P. Jain, A. S. Bradley, and C. W. Gardiner, Quantum de Laval nozzle: Stability and quantum dynamics of sonic horizons in a toroidally trapped Bose gas containing a superflow, Phys. Rev. A 76, 023617 (2007).

[47] J. Steinhauer, Observation of self-amplifying Hawking radiation in an analogue black-hole laser, Nat. Phys. 10, 864 (2014).

[48] J. Steinhauer and J. R. M. de Nova, Self-amplifying Hawking radiation and its background: A numerical study, Phys. Rev. A 95, 033604 (2017).

[49] S. Corley and T. Jacobson, Black hole lasers, Phys. Rev. D 59, 124011 (1999).

[50] Y.-H. Wang, T. Jacobson, M. Edwards, and C. Clark, Induced density correlations in a sonic black hole condensate, SciPost Phys. 3, 022 (2017).

[51] Y.-H. Wang, T. Jacobson, M. Edwards, and C. W. Clark, Mechanism of stimulated Hawking radiation in a laboratory Bose-Einstein condensate, Phys. Rev. A 96, 023616 (2017).

[52] J. R. M. de Nova, S. Finazzi, and I. Carusotto, Time-dependent study of a black-hole laser in a flowing atomic condensate, Phys. Rev. A 94, 043616 (2016).

[53] M. Visser, Acoustic black holes: Horizons, ergospheres and Hawking radiation, Classical Quantum Gravity 15, 1767 (1998).

[54] R. Balbinot, A. Fabbri, S. Fagnocchi, A. Recati, and I. Carusotto, Nonlocal density correlations as a signature of Hawking radiation from acoustic black holes, Phys. Rev. A 78, 021603(R) (2008).

[55] R. Parentani, From vacuum fluctuations across an event horizon to long distance correlations, Phys. Rev. D 82, 025008 (2010).

[56] J. Steinhauer, Measuring the entanglement of analogue Hawking radiation by the density-density correlation function, Phys. Rev. D 92, 024043 (2015).

[57] A. Sinatra, C. Lobo, and Y. Castin, Classical-Field Method for Time Dependent Bose-Einstein Condensed Gases, Phys. Rev. Lett. 87, 210404 (2001).

[58] A. Sinatra, C. Lobo, and Y. Castin, The truncated Wigner method for Bose-condensed gases: Limits of validity and applications, J. Phys. B: At. Mol. Opt. Phys. 35, 3599 (2002).

[59] J. Ruostekoski and A. D. Martin, The Truncated Wigner Method for Bose Gase (Imperial College Press, London, 2013), pp. 203-214.

[60] B. Opanchuk and P. D. Drummond, Functional Wigner representation of quantum dynamics of Bose-Einstein condensate, J. Math. Phys. 54, 042107 (2013).

[61] P. D. Drummond and B. Opanchuk, Truncated Wigner dynamics and conservation laws, Phys. Rev. A 96, 043616 (2017).
[62] A. D. Jackson, G. M. Kavoulakis, and C. J. Pethick, Solitary waves in clouds of Bose-Einstein condensed atoms, Phys. Rev. A 58, 2417 (1998).

[63] P. Leboeuf and N. Pavloff, Bose-Einstein beams: Coherent propagation through a guide, Phys. Rev. A 64, 033602 (2001).

[64] A. Muñoz Mateo and V. Delgado, Effective mean-field equations for cigar-shaped and disk-shaped Bose-Einstein condensates, Phys. Rev. A 77, 013617 (2008).

[65] P. L. Halkyard, M. P. A. Jones, and S. A. Gardiner, Rotational response of two-component Bose-Einstein condensates in ring traps, Phys. Rev. A 81, 061602(R) (2010).

[66] G. Theocharis, P. Schmelcher, P. G. Kevrekidis, and D. J. Frantzeskakis, Matter-wave solitons of collisionally inhomogeneous condensates, Phys. Rev. A 72, 033614 (2005).

[67] G. Theocharis, P. Schmelcher, P. G. Kevrekidis, and D. J. Frantzeskakis, Dynamical trapping and transmission of matterwave solitons in a collisionally inhomogeneous environment, Phys. Rev. A 74, 053614 (2006).

[68] F. Kh. Abdullaev and M. Salerno, Adiabatic compression of soliton matter waves, J. Phys. B: At. Mol. Opt. Phys. 36, 2851 (2003).

[69] S. De Nicola, B. A. Malomed, and R. Fedele, An effective potential for one-dimensional matter-wave solitons in an axially inhomogeneous trap, Phys. Lett. A 360, 164 (2006).

[70] G. P. Agrawal, Nonlinear Fiber Optics (Academic, New York, 1995)

[71] A. Recati, N. Pavloff, and I. Carusotto, Bogoliubov theory of acoustic Hawking radiation in Bose-Einstein condensates, Phys. Rev. A 80, 043603 (2009).

[72] C. Mayoral, A. Fabbri, and M. Rinaldi, Steplike discontinuities in Bose-Einstein condensates and Hawking radiation: Dispersion effects, Phys. Rev. D 83, 124047 (2011).

[73] A. Fabbri and C. Mayoral, Steplike discontinuities in BoseEinstein condensates and Hawking radiation: The hydrodynamic limit, Phys. Rev. D 83, 124016 (2011).

[74] J. Macher and R. Parentani, Black-hole radiation in BoseEinstein condensates, Phys. Rev. A 80, 043601 (2009).

[75] C. Barceló, A. Cano, L. J. Garay, and G. Jannes, Stability analysis of sonic horizons in Bose-Einstein condensates, Phys. Rev. D 74, 024008 (2006).

[76] L. Bergé, Wave collapse in physics: Principles and applications to light and plasma waves, Phys. Rep. 303, 259 (1998).

[77] J. MacGibbon, Can Planck-mass relics of evaporating black holes close the universe? Nature (London) 329, 308 (1987).

[78] B. Carr, F. Kühnel, and M. Sandstad, Primordial black holes as dark matter, Phys. Rev. D 94, 083504 (2016).

[79] B. J. Carr, K. Kohri, Y. Sendouda, and J. Yokoyama, Constraints on primordial black holes, Phys. Rev. D 94, 044029 (2016).

[80] B. V. Lehmann, C. Johnson, S. Profumo, and T. Schwemberger, Direct detection of primordial black hole relics as dark matter, J. Cosmol. Astropart. Phys. 2019, 046 (2019).

[81] J. A. de Freitas Pacheco and J. Silk, Primordial rotating black holes, Phys. Rev. D 101, 083022 (2020). 